\title{
Surgical solutions to orofacial problems
}

CPD

\section{David Sherring}

\section{Background}

Orofacial problems present frequently to primary care providers. Many of these problems have a surgical solution. Some may require minor procedures, while others require major maxillofacial surgery.

\section{Objective}

The purpose of this article is to illustrate how some common orofacial presentations can be investigated and solutions found in conjunction with oral and maxillofacial surgeons.

\section{Discussion}

This article outlines a method of approach for some of the issues with which patients present to their primary healthcare provider that may be resolved using skills and techniques of maxillofacial surgery.
THE LITERATURE SUGGESTS that many patients will seek advice from their primary healthcare providers for non-dental orofacial symptoms rather than from a dentist. ${ }^{1-3}$ The problems may range from 'simple' dental problems to complex issues requiring medical investigation and multidisciplinary management.

Patients (or their carers) may present with a number of different problems such as:

- 'I have trouble chewing food.'

- 'I don't like the way my face looks.'

- 'My snoring at night keeps my partner awake; I have to sleep in another room.'

- 'I am having a problem with my mouth and jaws that requires an operation.'

These problems require thoughtful orofacial investigation.

\section{Tooth loss and chewing problems}

Most adults have 32 teeth (including third molar teeth, which are frequently removed because of insufficient space and associated morbidity). ${ }^{4}$ Naturally, teeth in the maxilla and mandible must occlude to ensure adequate masticatory function.

Many people attend a dentist 'as a last resort' once a tooth has started aching. In many cases, this will lead to the loss of a tooth that might otherwise have been preserved had the patient attended earlier. This pattern of behaviour is common and will often lead to the wearing of a denture to replace the missing teeth. Denture-related

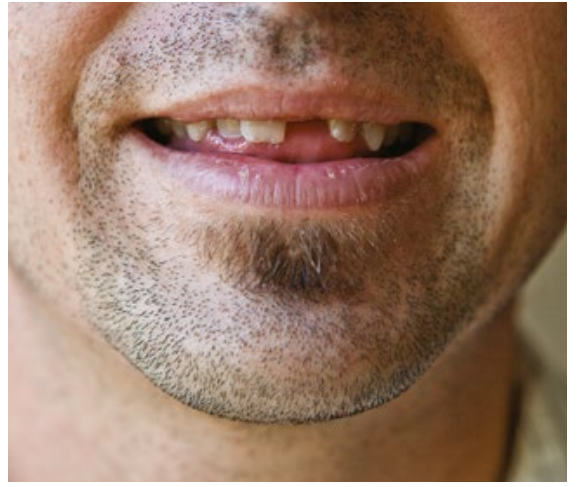

problems are another common cause of an inability to chew food satisfactorily.

Masticatory pain, related to temporomandibular joint dysfunction or other pain syndromes of the head and neck, may also result in the patient complaining of difficulty chewing. ${ }^{5}$ In these cases, referral to a general or specialist dentist should resolve the problem.

A patient presenting with unexpected weight loss or issues with gastrooesophageal reflux disease who assumes they have a masticatory problem presents a more complex issue. Assessment of dietary intake is important, as most people, even those that are without all their teeth (edentulous), are capable of maintaining weight on a soft, non-chew diet. Malnutrition may be associated with decreased chewing ability; however, there are many other causes that should be excluded prior to this diagnosis. ${ }^{6}$ Similarly, malignancy and neurological causes (among others) for unexplained weight loss should be investigated thoroughly.

Management of chewing problems in this group of patients may require multidisciplinary input from a speech pathologist, dietitian and/or physiotherapist to assess or manage the chewing problem.

Recent published studies have reported that the loss of as few as five teeth is associated with an increased incidence of acute myocardial infarction and 
other coronary heart disease events $\mathrm{s}^{7,8}$ and hypertension in postmenopausal women. ${ }^{9}$ Loss of more than nine teeth has been associated with incident cardiovascular disease, diabetes and all-cause mortality (Box 1$).{ }^{7}$ Patients with uncontrolled diabetes are prone to periodontitis, ${ }^{10}$ and this oral disease has an association with poor glycaemic control and microvascular complications. Periodontitis has been reported to be a strong predictor of cardiorenal mortality in this patient cohort with uncontrolled diabetes. ${ }^{11}$ Periodontitis is an inflammatory microvascular disease affecting the supporting structures of the dentition (periodontium and alveolar bone). ${ }^{12}$ This is the most common pathological process resulting in tooth loss in the ageing population. ${ }^{13}$

A recent cohort study reported a possible relationship between missing teeth and risk of dementia: the greater the number of missing teeth, the greater the risk of developing dementia. ${ }^{14}$ This finding has been supported by three previous meta-analyses. ${ }^{15-17}$ The authors discuss a number of possible mechanisms for this association, including altered chewing function, with patients wearing dentures to replace missing teeth at lower risk of developing dementia. The literature suggests masticatory function may promote blood supply to the brain ${ }^{18,19}$ and increased neurotransmitter secretion. ${ }^{20}$ Stabilisation of a mandibular denture with the insertion of two dental implant fixtures has been reported to improve brain function and cognitive function in edentulous patients. ${ }^{19}$

Care must be taken when correlating tooth loss with medical conditions in a direct cause-and-effect relationship.

Tooth loss in the elderly is primarily a result of dental caries, while periodontitis is a greater disease burden and more advanced in people aged $>65$ years. ${ }^{21}$ Both of these pathological processes are multifactorial but are often associated with poor oral hygiene and a dry mouth (xerostomia). Common causes of xerostomia include medication (both prescribed and over the counter) and drug abuse. ${ }^{22}$ A review of medication and referral for appropriate medical intervention is warranted, as is referral for restoration of the dentition. Concurrent referral to a dentist is appropriate in this situation (Figure 1).

Patients presenting with a chief complaint of tooth loss and chewing

Box 1. Comorbidities associated with missing teeth

- Acute myocardial infarction

- Coronary artery disease

- Hypertension

- Diabetes

- Dementia

- Malnutrition

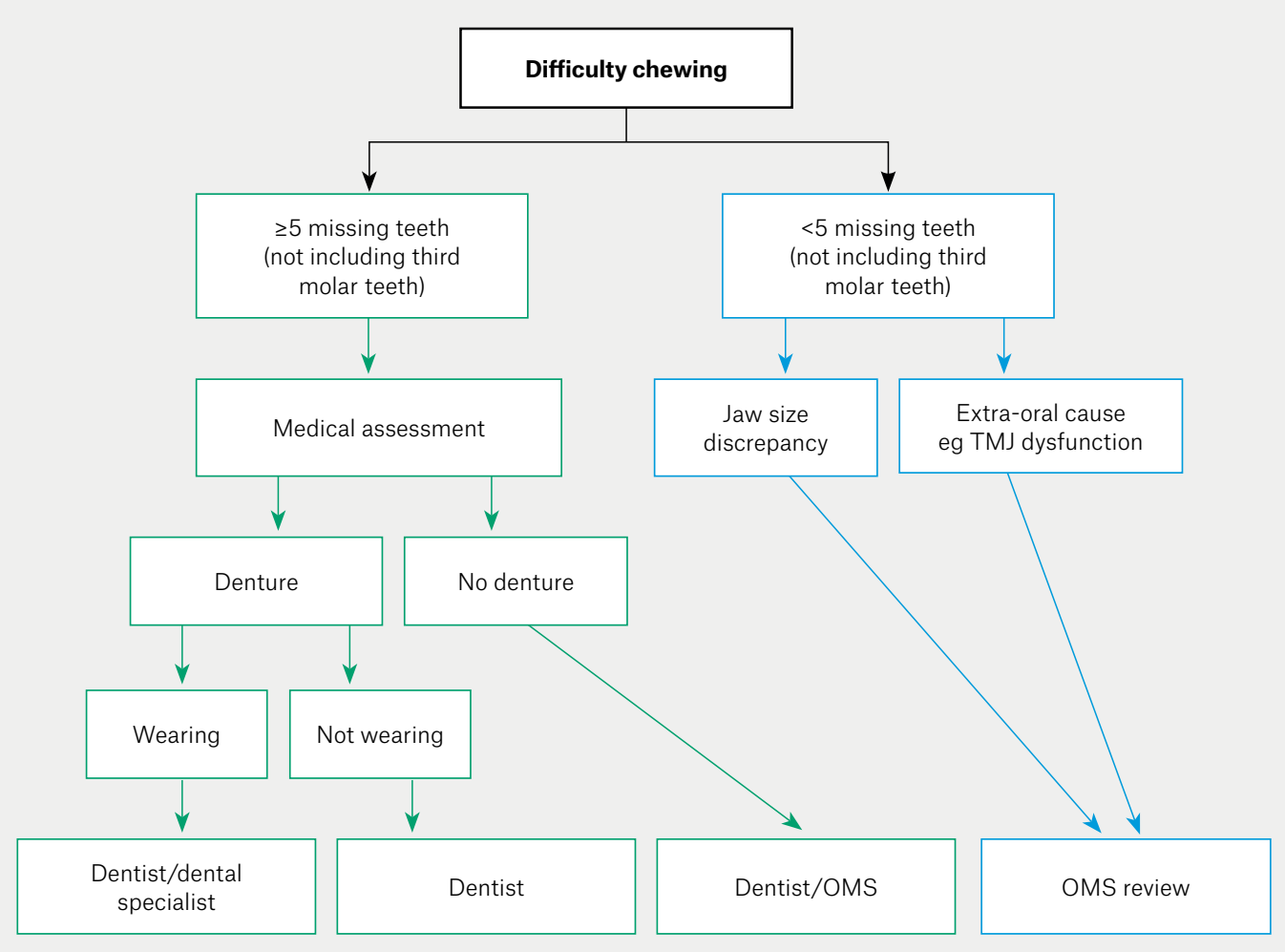

Figure 1. Suggested referral pattern for patients experiencing difficulty chewing

OMS, oral and maxillofacial surgeon; TMJ, temporomandibular joint 
problems frequently will be adequately

managed by referral to a dental

practitioner. Unfortunately, it is acknowledged that attendance may be hindered by a variety of factors including cost, long public waiting lists and fear. ${ }^{23}$ Patients may require medication or psychological counselling to overcome their fear of dental intervention.

Surgical options for patients with missing teeth or difficulty tolerating dentures range from relatively simple removal of excess soft tissue to facilitate better fit between the denture and underlying supporting tissues, to complex interventions to replace missing hard tissue with or without the use of dental implants.

Patients who are edentulous often have difficulty due to significant resorption of their alveolar bone (a normal process following tooth loss). Associated problems typically occur in the mandible, with neuropathic pain from pressure on the mental foramina, which are now at the crest of the edentulous ridge, or ulceration from pressure on the genial tubercles. In these cases, there is insufficient natural tissue for adequate denture support and function, and the use of two dental implants placed in the anterior mandible has been shown to significantly improve quality of life for such patients. Dental implants are titanium fixtures that form a biological attachment to the adjacent bone (osseointegration) and have been shown to have a success rate of $>96 \%$ at 10 years. ${ }^{24}$ The biological basis of the surgical technique has been described in detail elsewhere (Figure 2). ${ }^{25,26}$

\section{Skeletal jaw discrepancy and craniofacial syndromes}

Orofacial appearance helps define us, and deviation from societal norms can significantly adversely affect a patient's life and wellbeing. Problems with facial aesthetics related to hard tissue (bone and/or tooth) may be fundamentally caused by tooth loss, jaw malposition or the rare craniofacial syndrome.

Diagnosis of skeletal jaw discrepancy is generally undertaken by clinical and radiographic assessment against norms for sex and racial background. The primary care physician may be able to diagnose the patient clinically, particularly if there is a reverse overjet (the mandibular incisor teeth lie anterior to the maxillary incisor teeth), increased overjet (the maxillary incisor teeth lie $>5 \mathrm{~mm}$ anterior to the mandibular incisor teeth) or an open bite (the incisor teeth of the maxilla and mandible do not overlap). Referral to an orthodontist or oral and maxillofacial surgeon is indicated in these situations, as functional problems relating to masticatory function and speech, airway compromise and displeasing aesthetics are likely to be present.

Many of these patients can be adequately managed using orthodontics alone; however, approximately $5 \%$ of the population have a discrepancy in jaw position such that orthodontics alone cannot correct the problem. ${ }^{27}$ Further information regarding diagnosis, treatment planning and surgical techniques is available in a recent review (Figure 3). ${ }^{28}$

\section{Snoring and obstructive sleep apnoea}

Obstructive sleep apnoea (OSA) is becoming more prevalent in Australia as levels of obesity increase. ${ }^{29}$ Management of OSA is primarily aimed at lifestyle modification and the use of nasal continuous positive airway pressure (nCPAP) or a mandibular repositioning appliance. For patients with OSA who are not obese, the facial skeletal structure should be investigated, as jaw malposition is often the underlying cause of airway narrowing and obstruction during sleep. Facial osteotomies to correct jaw malposition have been confirmed to be as effective as nCPAP in treating OSA by increasing upper airway dimensions and decreasing resistance to airflow. ${ }^{30}$ This is in clear distinction to other surgical modalities such as uvulopalatopharyngoplasty (UPPP), tongue reduction surgery and other surgery removing bulky soft tissues. A meta-analysis published in 2010 found maxillomandibular advancement surgery either as a standalone procedure or as an adjunct to soft tissue procedures resulted in an Apnoea Hypopnoea Index (AHI)
$<10$ events/hour post-operatively. This is in contrast to UPPP (AHI averaging 29 events/hour post-operatively), modified UPPP and other pharyngeal procedures, which all resulted in an AHI averaging $>14$ events/hour post-operatively. ${ }^{31}$

Oral and maxillofacial surgeons work in close conjunction with respiratory physicians and, in a select population of people with OSA, are able to offer long-term effective treatment resulting in the removal of the requirement for nCPAP or other devices. Untreated or poorly treated OSA results in significant patient morbidity and premature mortality (Table 1). ${ }^{32}$

Craniofacial syndromes, including cleft lip and palate, are relatively uncommon. They present complex problems, and referral to a tertiary hospital for multidisciplinary assessment and treatment is required. These multidisciplinary teams will comprise oral and maxillofacial, plastic and ear, nose and throat surgeons. Other professionals including paediatric dentists, orthodontists, paediatricians, social workers and speech pathologists will also be involved. ${ }^{33,34}$

\section{Head and neck cancer}

Head and neck cancers are the seventh most commonly diagnosed cancers in Australia. ${ }^{35}$ Primary healthcare providers are key in ensuring patients presenting with head and neck cancers are adequately supported throughout their treatment and beyond.

The differential diagnosis of the numerous pathologies and dysfunctions of the orofacial region has been presented

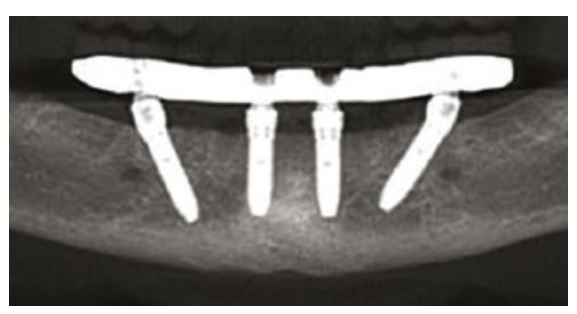

Figure 2. Standard mandibular 'all-on-4' implants ${ }^{26}$ 
elsewhere. ${ }^{5,25,26,28,30,33,36,37}$ Any background medical issues or issues related to treatment need to be monitored and managed where possible by the primary healthcare provider in concert with other specialties as needed. ${ }^{38}$

Treatment for head and neck cancers produces significant morbidity in survivors irrespective of the treatment modality (ie ablative surgery, radiation therapy, chemotherapy, immunotherapy or any combination of these).

Ablative surgery for the management of oral cancers should also include reconstruction of the defect to facilitate restoration of chewing function, which is vitally important for maintenance of adequate nutrition and also contributes to improved speech and swallowing. Patients presenting with issues related to oral function, particularly following treatment of head and neck cancers, should be reviewed by an oral and maxillofacial surgeon and speech pathologist. Options for reconstruction range from simple restoration of missing teeth to complex free tissue transfer with or without an implant-supported prosthesis. ${ }^{37}$ Composite flap reconstructions that are fully vascularised are often planned using virtual surgical planning, and three-dimensional surgical guides are produced (Figure 4). The available techniques are presented in detail in a recent publication. ${ }^{39}$

Radiation therapy with or without concomitant chemotherapy or immunotherapy may present acute problems during treatment such as mucositis and pain resulting in an impaired ability to eat. Long-term problems generally present secondary to salivary gland dysfunction and fibrosis. These glands are particularly radiosensitive and become non-functional following radiation for head and neck cancers if they are in the field of radiation. Patients may present with a dry mouth (xerostomia), difficulties chewing and swallowing, disturbed taste, dental caries, an inability to tolerate dentures ${ }^{38}$ and osteoradionecrosis (Table 2). At 12 months following diagnosis, quality of life related to eating has been reported to be the most significant physical issue experienced by survivors of treatment for head and neck cancers. ${ }^{40}$

Patients presenting with issues related to a dry mouth should be encouraged to avoid snacking and sugary foods. It is beneficial to these patients to frequently sip water and to consult with their
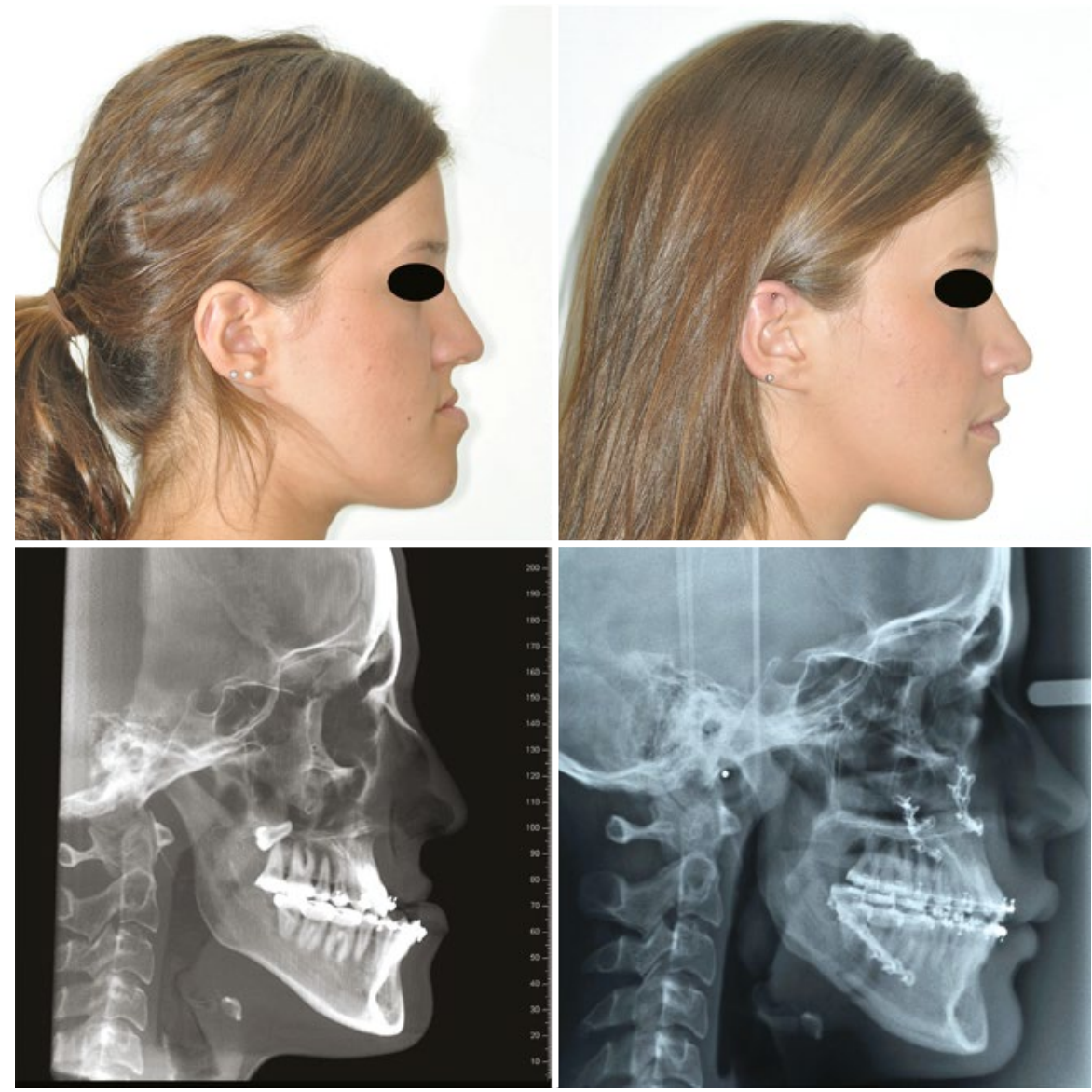

Figure 3. Small maxilla and large mandible treated by maxillary advancement and mandibular setback ${ }^{28}$

\section{Table 1. Medical issues related to obstructive sleep apnoea}

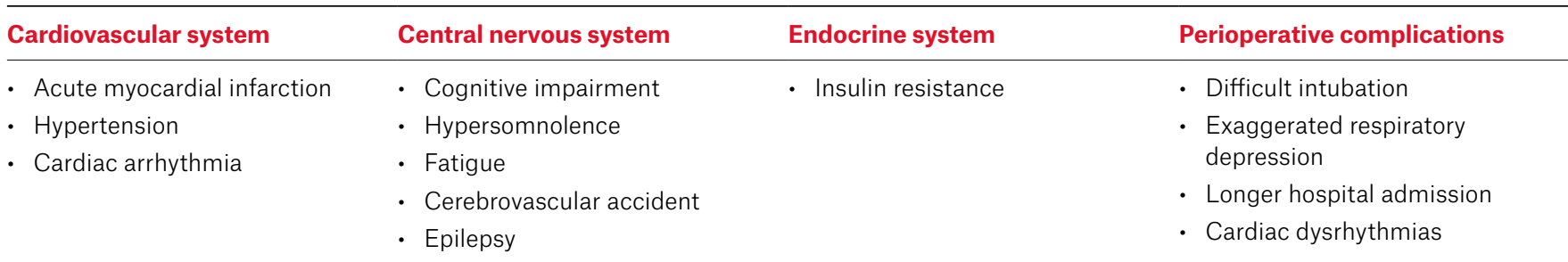


dentists at least every three months, as dental decay and mucosal issues such as candidiasis are more frequent and progress rapidly in this group of patients.

Dentures to replace missing teeth in patients who have received radiation therapy are contraindicated in the field of radiation if mucosal supported because of the risk of provoking osteoradionecrosis. In this situation, assessment by an oral and maxillofacial surgeon and prosthodontist may allow formulation of a treatment plan whereby the prostheses can be supported by dental implants placed in non-irradiated bone. This will require liaison between the oral and maxillofacial surgeon and radiation oncologist to determine the field of exposure.

Medicare Benefits Schedule (MBS) item numbers are available to oral and

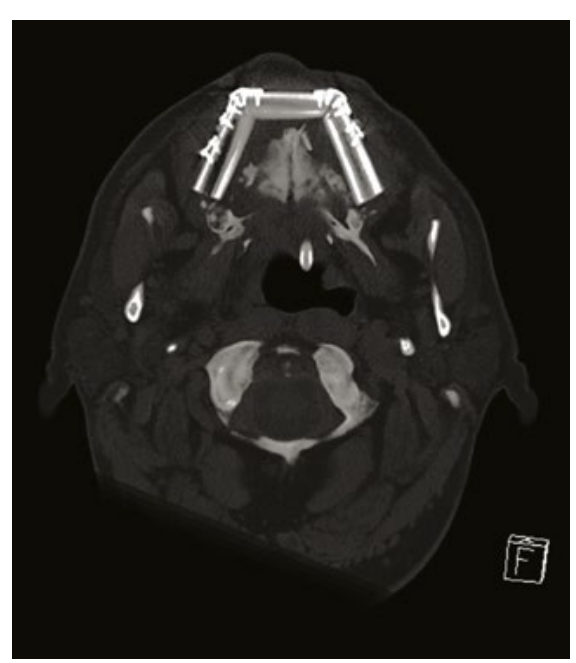

Figure 4. Osteotomies of a fibula free flap to reconstruct a maxilla ${ }^{39}$

\section{Table 2. Common oral problems following head and neck radiotherapy}

\begin{tabular}{ll}
\hline Acute & Chronic \\
\hline - Mucosal inflammation (mucositis) & $\cdot$ Dry mouth (xerostomia) \\
- Oral/pharyngeal ulceration & $\cdot$ Altered taste (dysgeusia) \\
- Candidiasis & $\cdot$ Difficulty swallowing \\
& $\cdot$ Dental decay (caries) \\
& $\cdot$ Candidiasis \\
& $\cdot$ Difficulty tolerating dentures \\
& $\cdot$ Osteoradionecrosis \\
\hline
\end{tabular}

maxillofacial surgeons who place dental implants in patients who have undergone treatment for head and neck malignancy, significantly decreasing the cost to the patient. However, there remain issues with provision of dental prostheses, with public subsidy being very limited and varying between states and territories. Non-cancer cases are not funded by the MBS.

\section{Conclusion}

The management of patients presenting with chewing difficulties, jaw malalignment or side effects related to head and neck cancers is challenging, often requiring multidisciplinary input. The initial presentation may be complex, and it is important to consider functional issues in these patient groups to assist with improving quality of life. Surgery may be an option for some patients to resolve their presenting complaint.

\section{Author}

David Sherring BDS, MBBS, DClinD,

FRACDS (OMS), Adjunct Associate Professor, Oral and Maxillofacial Surgery, Charles Sturt University, NSW.sherringd@hotmail.com

Competing interests: None.

Funding: None.

Provenance and peer review: Commissioned, externally peer reviewed.

\section{References}

1. Bell GW, Smith GLF, Rodgers JM, Flynn RW, Malone $\mathrm{CH}$. Patient choice of primary care practitioners for orofacial symptoms. Br Dent J 2008;204(12):669-73. doi: 10.1038/ sj.bdj.2008.523.

2. Mansour MH, Cox SC. Patients presenting to the general practitioner with pain of dental origin. Med J Aust 2006;185(2):64-67. doi: 10.5694/j.13265377.2006.tb00472.x

3. Lockhart PB, Mason DK, Konen JC, Gibson J. Prevalence and nature of orofacial and dental problems in family medicine. Arch Fam Med 2000;9(10):1009-12. doi: 10.1001/ archfami.9.10.1009.

4. Hyam DM. The contemporary management of third molars. Aust Dent J 2018;63 Suppl 1:S19-S26. doi: 10.1111/adj.12587.

5. Lomas J, Gurgenci T, Jackson C, Campbell D. Temporomandibular dysfunction. Aust J Gen Pract 2018;47(4):212-15. doi: 10.31128/AFP-10-17-4375.

6. Visvanathan R, Newbury JW, Chapman I. Malnutrition in older people - Screening and management strategies. Aust Fam Physician 2004;33(10):799-805.

7. Liljestrand JM, Havulinna AS, Paju S, Männistö S, Salomaa V, Pussinen PJ. Missing teeth predict incident cardiovascular events, diabetes and death. J Dent Res 2015;94(8):1055-62. doi: 10.1177/0022034515586352.

8. Abnet CC, Qiao YL, Dawsey SM, Dong ZW, Taylor PR, Mark SD. Tooth loss is associated with increased risk of total death and death from upper gastrointestinal cancer, heart disease, and stroke in a Chinese population-based cohort. Int J Epidemiol 2005;34(2):467-74. doi: 10.1093/ije/ dyh375.

9. Taguchi A, Sanada M, Suei Y, et al. Tooth loss is associated with an increased risk of hypertension in postmenopausal women. Hypertension 2004:43(6):1297-300. doi: 10.1161/01. HYP.0000128335.45571.ce.

10. Taylor JJ, Preshaw PM, Lalla E. A review of the evidence for pathogenic mechanisms that may link periodontitis and diabetes. J Periodontol 2013;84(4 Suppl):S113-S34. doi: 10.1902/ jop.2013.134005

11. Saremi A, Nelson RG, Tulloch-Reid M, et al. Periodontal disease and mortality in type 2 diabetes. Diabetes Care 2005;28(1):27-32. doi: 10.2337/diacare.28.1.27.

12. Highfield J. Diagnosis and classification of periodontal disease. Aust Dent J 2009;54 Suppl 1:S11-S26. doi: 10.1111/j.1834-7819.2009.01140.x.

13. Gonda T, MacEntee MI, Kiyak HA, Persson GR, Persson RE, Wyatt C. Predictors of multiple tooth loss among socioculturally diverse elderly subjects. Int J Prosthodont 2013;26(2):127-34. doi: 10.11607/ ijp.2893.

14. Chen J, Ren CJ, Wu L, et al. Tooth loss is associated with increased risk of dementia and with a dose-response relationship. Front Aging Neurosci 2018;10:415. doi: 10.3389/ fnagi.2018.00415.

15. Shen T, Lv J, Wang L, Wang W, Zhang D. Association between tooth loss and dementia among older people: A meta-analysis. Int J Geratr Psychiatry 2016;31(8):953-55. doi: 10.1002/ gps.4396.

16. Tonsekar PP, Jiang SS, Yue G. Periodontal disease, tooth loss and dementia: Is there a link? A systematic review. Gerodontology 2017;34(2):151-63. doi: 10.1111/ger.12261.

17. Oh $\mathrm{B}, \mathrm{Han} \mathrm{DH}, \mathrm{Han} \mathrm{KT}$, et al. Association between residual teeth number in later life and incidence of dementia: A systematic review and meta-analysis. BMC Geriatr 2018;18(1):48. doi: 10.1186/s12877018-0729-z.

18. Yamamoto T, Kondo K, Hirai H, Nakade M, Aida $J$, Hirata Y. Association between self-reported dental health status and onset of dementia: A 4-year prospective cohort study of older Japanese adults from the Aichi Gerontological Evaluation Study (AGES) project. Psychosom Med 2012;74(3):241-48. doi: 10.1097/ PSY.0b013e318246dffb. 
19. Banu RF, Veeravalli PT, Kumar VA. Comparative evaluation of changes in brain activity and cognitive function of edentulous patients, with dentures and two-implant supported mandibular overdenture-pilot study. Clin Implant Dent Relat Res 2016;18(3):580-87. doi: 10.1111/cid.12336.

20. Lin CS. Revisiting the link between cognitive decline and masticatory dysfunction. BMC Geriartr 2018;18(1):5. doi: 10.1186/s12877-0170693-z.

21. Australian Institute of Health and Welfare. Older Australia at a glance. Cat. no. AGE 87. Canberra, ACT: AlHW, 2018. Available at www.aihw.gov.au/ reports/older-people/older-australia-at-a-glance [Accessed 12 August 2020].

22. Tanasiewicz M, Hildebrandt T, Obersztyn I. Xerostomia of various etiologies: A review of the literature. Adv Clin Exp Med 2016;25(1):199-206. doi: 10.17219/acem/29375.

23. Marshall RI, Spencer AJ. Accessing oral health care in Australia. Med J Aust 2006;185(2):59-60. doi: 10.5694/j.1326-5377.2006.tb00470.x

24. How MS, Keys W, Richards D. Long-term (10-year) dental implant survival: A systematic review and sensitivity meta-analysis. J Dent 2019;84:9-21. doi: 10.1016/j.jdent.2019.03.008.

25. Ruljancich K. Implantology in oral and maxillofacial surgery. The compexity of 'simple' cases. Aust Dent J 2018;63(S1):S27-S34. doi: 10.1111/adj.12588.
26. Spencer KR. Implant based rehabilitation options for the atrophic edentulous jaw. Aust Dent J 2018;63 Suppl 1:S100-S07. doi: 10.1111/adj.12595.

27. Proffit WR, White RP Jr. Combined surgicalorthodontic treatment: How did it evolve and what are the best practices now? Am J Orthod Dentofacial Orthop 2015;147(5 Suppl):S205-15. doi: 10.1016/j.ajodo.2015.02.009.

28. Lim L, Heggie A. Versatile facial osteotomies. Aust Dent J 2018;63 Suppl 1:S48-S57. doi: 10.1111/ adj.12590.

29. Garvey JF, Pengo MF, Drakatos P, Kent BD. Epidemiological aspects of obstructive sleep apnea. J Thorac Dis 2015;7(5):920-29. doi: 10.3978/j.issn.2072-1439.2015.04.52.

30. Holty JEC, Guilleminault C. Maxillomandibular advancement for the treatment of obstructive sleep apnea: A systematic review and metaanalysis. Sleep Med Rev 2010;14(5):287-97. doi: 10.1016/j.smrv.2009.11.003.

31. Caples SM, Rowley JA, Prinsell JR. Surgical modifications of the upper airway for obstructive sleep apnea in adults: A systematic review and meta-analysis. Sleep 2010;33(10):1396-407. doi: 10.1093/sleep/33.10.1396.

32. Park JG, Ramar K, Olson EJ. Updates on definition, consequences, and management of obstructive sleep apnea. Mayo Clin Proc 2011;86(6):554-55. doi: 10.4065/mcp.2010.0810.
33. Heggie AA. Craniofacial disorders. Aust Dent $J$ 2018;63(S1):S58-S68. doi: 10.1111/adj.12591.

34. Shand JM. Paediatric oral \& maxillofacial surgery. Aust Dent J 2018;63 Suppl 1:S69-S78. doi: 10.1111/ adj.12592.

35. National Cancer Control Indicators. Cancer Incidence. Surry Hills, NSW: NCCl, 2019. Available at https://ncci.canceraustralia.gov.au/diagnosis/ cancer-incidence/cancer-incidence [Accessed 22 June 2020].

36. Wong T, Yap T, Wiesenfeld D. Common benign and malignant oral mucosal disease. Aust J Gen Pract 2020;49(9):568-73.

37. Wong T, Yap T, Wiesenfeld D. Common causes of 'swelling' in the oral cavity. Aust J Gen Pract 2020;49(9):575-80.

38. Koh J, Walsh P, D'Costa I, Bhatti O. Head and neck squamous cell carcinoma survivorship care. Aust J Gen Pract 2019;48(12):846-48. doi: 10.31128/ AJGP-08-19-5032.

39. Batstone MD. Reconstruction of major defects of the jaws. Aut Dent J 2018;63(S1):S108-S13. doi: 10.1111/adj.12596.

40. Ronis DI, Duffy SA, Fowler KE, Khan MJ, Terrell JE. Changes in quality of life over 1 year in head and cancer. Arch Otolaryngol Head Neck Surg 2008;134(3):241-48. doi: 10.1001/ archoto.2007.43. 